\title{
EDITORIAL
}

\section{PROFESIONALES DE ENFERMERÍA Y EL CUIDADO DE MIGRANTES INTERNACIONALES EN CHILE: ROL DE LA COMPETENCIA CULTURAL}

\author{
NURSING PROFESSIONALS AND THE CARE OF \\ INTERNATIONAL MIGRANTS IN CHILE: ROLE OF CULTURAL \\ COMPETITION
}

\author{
Marcela Correa-Betancour, Enfermera \\ Departamento de Enfermería, Universidad de Chile, \\ Santiago, Chile
}

E-mail: marcelacorrea@uchile.cl

La movilidad humana ha acompańado la vida en sociedad desde el inicio de los tiempos. Es así que la migración internacional no es un fenómeno reciente, sino más bien existen elementos del contexto actual que configuran un escenario más complejo que en el pasado, pero que es conocido en sus raíces y motivaciones.

En Chile la llegada de migrantes internacionales ha sido variable en el tiempo ${ }^{(1)}$, sin embargo, lo que comienza a experimentar el país desde el año 2002 y especialmente desde 2010, no tiene precedente en su historia. El escenario sociodemográfico actual indica que en Chile existen aproximadamente 1,2 millones de migrantes internacionales, y de ellos, más del $60 \%$ llegó después de $2010^{(2,3)}$. Esta cifra representa aproximadamente un 4,4\% de la población residente en el país, trayendo desafíos no solo a los migrantes y sus familias sino también a la sociedad chilena y a las instituciones sociales que han debido generar mecanismos para dar respuesta a esta nueva realidad.

En el área de la salud se han levantado múltiples iniciativas destinadas a fomentar la integración de personas migrantes al sistema y garantizar el acceso a la salud de ellos y sus familias, asumiendo la salud como un derecho humano básico. Muestra de ello es la presencia de facilitadores interculturales en muchos centros de atención primaria y terciaria, la traducción de material clínico e informativo, adaptación de pautas e indicaciones nutricionales, así como la puesta en vigencia de la circular A1504 del 13 de junio de 2016, que garantiza atención de salud a toda persona independiente de su condición migratoria ${ }^{(4)}$. Es de suponer que el profesional de enfermería ha tenido un rol protagónico en el desarrollo e implementación de muchas de estas medidas, pues en su labor clínica se vincula directamente con estas comunidades, realizando esfuerzos tendientes a lograr una atención de salud pertinente a los migrantes internacionales. Aun así, hasta ahora se evidencia una brecha en la formación del 
profesional de enfermería respecto a cómo abordar la gestión del cuidado de personas, familias y comunidades que provengan de diferentes culturas ${ }^{(5,6)}$.

Para el abordaje de este vacío sería necesario promover en los profesionales de enfermería el desarrollo de la denominada competencia cultural que es definida como un conjunto de habilidades, conocimientos y aptitudes, e incluso políticas, que habilitan al profesional de salud, en este caso de enfermería, para trabajar de forma eficiente con personas, familias y comunidades de diferente origen cultural ${ }^{(7)}$.

La evidencia, principalmente emanada de países con alta proporción de migrantes internacionales, es clara al demostrar la mejora significativa de los indicadores de salud en aquellas comunidades en donde se ha adoptado como una estrategia la capacitación del profesional de enfermería con la competencia cultural para trabajar con grupos minoritarios como los inmigrantes. Ejemplo de ello se encuentra en los trabajos de Betancourt et al. ${ }^{(8,9)} \mathrm{o}$ Bruhn $^{(10)}$. Visto esto y reconociendo la existencia de múltiples culturas dentro de nuestro país, además de las vinculadas con colectivos migrantes, es que la formación de los profesionales de enfermería debiese incluir de forma explícita la capacitación en competencia cultural, con el objetivo de habilitarles a trabajar de forma respetuosa, efectiva, culturalmente pertinente y manteniendo estándares de calidad en diferentes contextos culturales.

Ante el nuevo escenario sociodemográfico, la formación en competencia cultural de los profesionales de enfermería debería abordarse desde distintos ámbitos, a saber: la incorporación temprana de estos contenidos en las mallas curriculares de enfermería como elemento transversal de la formación; capacitación y sensibilización individual de los profesionales de enfermería ya titulados que se encuentren insertos en el sistema de salud público o privado, independiente de su labor (clínica, administrativa, gestión, educación, política); y finalmente, incorporación de la competencia cultural como política de calidad en las instituciones de salud públicas y privadas. Estas medidas permitirían reconocer el impacto de la capacitación en competencia cultural en la salud de las personas migrantes internacionales, responder a sus necesidades de salud de forma adecuada, ética, respetuosa e individualizada, garantizando el derecho a la salud de todos quienes residen en el territorio.

Un profesional de enfermería capacitado en competencia cultural será capaz de abordar de mejor manera las necesidades de salud de la sociedad actual que se desarrolla en un mundo globalizado y dinámico. Finalmente, se destaca la emergente necesidad de generar futuras investigaciones que identifiquen y permitan abordar las brechas de formación en los profesionales de enfermería, así como la realización de estudios que permitan cuantificar el impacto de la formación de profesionales de enfermería con competencia cultural en la salud de los migrantes internacionales y los grupos minoritarios presentes en nuestra sociedad.

\section{REFERENCIAS}

1. Stefoni C. Perfil Migratorio de Chile [Internet]. Argentina: International Organization for Migration; 2011 [citado 30 oct 2019]. 109 p. Disponible en: http://incami.cl/wpcontent/uploads/2013/05/LIBRO-OIM-PERFILMIGRATORIODECHILE-2011.pdf

2. Instituto nacional de estadísticas. Síntesis de Resultados CENSO 2017 [Internet]. Santiago; 2018 [citado 30 oct 2019]. 27 p. Disponible en: https://www.censo2017.cl/ descargas/home/sintesis-de-resultados-censo2017.pdf 
3. Instituto Nacional de Estadísticas. Departamento de Extranjería y Migración. Estimación de Personas Extranjeras Residentes en Chile, 31 diciembre 2018 [Internet]. Santiago; 2019 [citado el 30-10-2019]. 24 p. Disponible en: https://www.extranjeria.gob.cl/media/2019/04/ Presentaci\%C3\%B3n-Extranjeros Residentes-en-Chile.-31-Diciembre-2018.pdf

4. Correa M. Migraciones y políticas de salud en Chile: cronología de los principales hitos 2000-2018. A\&C [Internet]. 2018 [citado 30 oct 2019]; 2(1): 210-226. Disponible en: http://revistas.academia.cl/index.php/academiaycritica/article/view/886/1111

5. Correa M, Carstens C, Reyes S. Experiencia del diseño e implementación de un Curso de Formación General de Migración y Salud en la Facultad de Medicina de la Universidad de Chile. Cuadernos Médicos y Sociales. 2018 [citado 30 oct 2019]; 58(4): 55-63.

6. Bernales M, Pedrero V, Obach A, Pérez C. Competencia Cultural en Salud: Una necesidad urgente en trabajadores de la salud. Rev Med Chile [Internet]. 2015 [citado 30 oct 2019]; 143(3): 401-402. Disponible en: https://doi.org/10.4067/S003498872015000300018

7. Jongen C, McCalman J, Bainbridge R, Clifford A. Cultural Competence in Health: A Review of the Evidence [Internet]. Springer Singapore; 2017 [citado 30 oct 2019]. 139 p. Disponible en: https://books.google.co.uk/books?id=E-85DwAAQBAJ

8. Betancourt JR, Duong JV, Bondaryk MR. Strategies to Reduce Diabetes Disparities: An Update. Curr Diab Rep [Internet]. 2012 [citado 30 oct 2019]; 12(6): 762-768. Disponible en: https://doi.org/10.1007/s11892-012-0324-1

9. Betancourt JR, Green AR, Carrillo JE, Park ER. Cultural Competence and Health Care Disparities: Key Perspectives and Trends. Health Aff [Internet]. 2005 [citado 30 oct 2019]; 24(2): 499-506. Disponible en: https://doi.org/10.1377/hlthaff.24.2.499

10. Bruhn JG. Culture and Health Disparities Evaluation of interventions and outcomes in the U.S. - Mexico border region. $1^{\text {era }}$ ed. Arizona: Springer International Publishing; 2014. 83p. 